\title{
Peripheral blood markers predictive of outcome and immune-related adverse events in advanced non-small cell lung cancer treated with PD-1 inhibitors
}

\author{
Lihong Peng ${ }^{1} \cdot$ Yong Wang $^{1,2} \cdot$ Fen Liu $^{3} \cdot$ Xiaotong Qiu $^{1} \cdot$ Xinwei Zhang $^{1} \cdot$ Chen Fang $^{1} \cdot$ Xiaoyin Qian $^{1} \cdot$ Yong Li $^{1}$ (D)
}

Received: 17 December 2019 / Accepted: 17 April 2020 / Published online: 29 April 2020

(c) The Author(s) 2020

\begin{abstract}
Background Selected patients with advanced non-small cell lung cancer (NSCLC) benefit from immunotherapy, especially immune checkpoint inhibitors such as PD-1 (programmed cell death protein 1) inhibitor. Peripheral blood biomarkers would be most convenient to predict treatment outcome and immune-related adverse events (irAEs) in candidate patients. This study explored associations between inflammation-related peripheral blood markers and onset of irAEs and outcome in patients with advanced NSCLC receiving PD-1 inhibitors.

Methods A retrospective analysis was conducted of 102 patients with advanced NSCLC receiving PD-1 inhibitors from January 2017 to May 2019. Cox regression models were employed to assess the prognostic effect of low/high neutrophil/ lymphocyte ratio (NLR), lactate dehydrogenase (LDH), and prognostic nutrition index (PNI) on overall survival (OS) and progression-free survival (PFS). Logistic regression models were used to analyze the correlation between peripheral blood markers and the onset of irAEs.

Result NLR $<5, \mathrm{LDH}<240 \mathrm{U} / \mathrm{L}$, or PNI $\geq 45$ was favorably associated with significantly better outcomes compared with higher, higher, or lower values, respectively. The multivariate analysis determined that these parameters were independently associated with both better PFS ( $p=0.049,0.046,0.014$, respectively) and longer OS ( $p=0.007,0.031,<0.001$, respectively). Patients with three favorable factors among NLR, LDH, and PNI had better PFS and OS than did those with two, one, or none. PNI and NLR were associated with the onset of irAEs.

Conclusion In patients with advanced NSCLC treated with PD-1 inhibitors, pretreatment NLR, LDH, and PNI may be useful predictive markers of clinical outcome and irAEs.
\end{abstract}

Keywords Lung cancer $\cdot$ Immunotherapy $\cdot$ Immune-related adverse events $\cdot$ Neutrophil-to-lymphocyte ratio $\cdot$ Lactate dehydrogenase $\cdot$ Prognostic nutrition index

Lihong Peng and Yong Wang have contributed equally to this work.

Yong $\mathrm{Li}$

liyongcsco@email.ncu.edu.cn

1 Department of Medical Oncology, First Affiliated Hospital of Nanchang University, 17 Yongwai Zheng Road, Nanchang 330000, China

2 Department of Medical Oncology, Affiliated Ganzhou Hospital of Nanchang University (Ganzhou People's Hospital), 18 Meiguan Road, Ganzhou 341000, China

3 Critical Care Medicine, First Affiliated Hospital of Nanchang University, 17 Yongwai Zheng Road, Nanchang 330000, China

$\begin{array}{ll}\text { Abbreviations } \\ \text { AE } & \text { Adverse event } \\ \text { ALK } & \text { Anaplastic lymphoma kinase } \\ \text { CI } & \text { Confidence interval } \\ \text { CT } & \text { Computed tomography } \\ \text { ECOG PS } & \text { Eastern Cooperative Oncology Group perfor- } \\ & \text { mance status } \\ \text { EGFR } & \text { Epidermal growth factor receptor } \\ \text { HR } & \text { Hazard ratio } \\ \text { irAEs } & \text { Immune-related adverse events } \\ \text { LDH } & \text { Lactate dehydrogenase } \\ \text { MSI-H } & \text { Microsatellite instability-high } \\ \text { NLR } & \text { Neutrophil-to-lymphocyte ratio } \\ \text { NR } & \text { Not reached } \\ \text { NSCLC } & \text { Non-small cell lung cancer } \\ \text { OR } & \text { Odds ratio }\end{array}$




$\begin{array}{ll}\text { OS } & \text { Overall survival } \\ \text { PD-1 } & \text { Programmed cell death-1 } \\ \text { PD-L1 } & \text { Programmed death-ligand 1 } \\ \text { PFS } & \text { Progression-free survival } \\ \text { PNI } & \text { Prognostic nutrition index } \\ \text { ROS1 } & \text { c-ros oncogene 1 } \\ \text { TMB } & \text { Tumor mutational burden }\end{array}$

\section{Introduction}

Whether across the globe or in China, lung cancer is the primary cause of cancer-related deaths [1]. Non-small cell lung cancer (NSCLC) accounts for $~ 85 \%$ of cases, and a majority include distant metastasis at diagnosis.

In recent years, immunotherapy has been associated with improved long-term survival in advanced NSCLC. The treatment options of patients have vastly expanded, especially through the use immune checkpoint inhibitors [2]. However, only a minority of patients have benefited [3]. There are predictive biomarkers that may be helpful for selecting patients for immunotherapy. Among the most recently recognized are PD-L1 (programmed death-ligand 1), TMB (tumor mutational burden), and MSI-H (microsatellite instability-high). However, these markers are costly, and the testing technology is not yet mature. On the other hand, if peripheral blood markers were known, the test would be clinically convenient and practically noninvasive.

Inflammation is closely linked to cancer, as it promotes a favorable microenvironment for cancer cell growth and spread, and activation of carcinogenic signaling pathways [4]. The prognostic value of some inflammation-related peripheral blood parameters has been investigated, including the neutrophil-to-lymphocyte ratio (NLR), lactate dehydrogenase (LDH), and prognostic nutritional index (PNI). Calculation of the NLR depends on the absolute neutrophil count and the absolute lymphocyte count within the peripheral blood; some studies have shown that NLR is associated with worsened prognosis in patients with melanoma receiving immunotherapy [5-7]. Similarly, a high pretreatment NLR is putatively a poor prognostic indicator in NSCLC [8-10]. So too, high serum LDH is reportedly a risk factor of poor prognosis in patients with NSCLC [11-13].

The PNI is based on serum albumin level and total lymphocyte count. It is easily calculated in daily routine, and a simple means to assess perioperative immunological and nutritional condition and stratify the risk of postoperative complications [14]. The preoperative or pretreatment PNI status is a good prognostic indicator in various cancers, including lung [15, 16], gastric [17], and colorectal [18] cancers, and glioblastoma [19]. However, sufficient data regarding the application of the PNI in the field of cancer immunotherapy are lacking.
Although patients can better tolerate immune checkpoint inhibitors compared with traditional chemotherapy, some patients still suffer adverse events which were known as immune-related adverse events (irAEs) that may cause treatment discontinuation or even death. Markers that may predict the onset of irAEs are unknown.

This retrospective study explored associations between inflammation-related peripheral blood markers (NLR, LDH, and PNI), and outcome and onset of irAEs in patients with advanced NSCLC receiving PD-1 inhibitors.

\section{Materials and methods}

\section{Patients}

The Research Ethics Board of First Affiliated Hospital of Nanchang University approved this retrospective study. The study population comprised 102 patients with a histologically or cytologically proven diagnosis of advanced NSCLC (IIIB/IV), who were treated with anti-PD-1 antibodies at our hospital from January 2017 to May 2019. Further inclusion criteria were an Eastern Cooperative Oncology Group performance status (ECOG PS) of 0-2, and $\geq 4$ cycles of immunotherapy treatment. Patients with any of the following were excluded from this study: autoimmune disease; pulmonary interstitial disease; adrenal insufficiency; or systemic immunosuppression.

\section{Treatment and data collection}

Patients received the following, until tumor progression, development of unacceptable drug toxicity, withdrawal, or death: nivolumab and toripalimab (intravenously $3 \mathrm{mg} / \mathrm{kg}$ every 2 weeks); sintilimab (200 mg every 3 weeks); and pembrolizumab ( $2 \mathrm{mg} / \mathrm{kg}$ every 3 weeks). The patients' clinical characteristics were collected, including age, gender, histology, sensitive gene mutation status and so on.

Baseline measurements were defined as those taken within 1 week before receiving PD-1 inhibitors. The baseline peripheral blood data including absolute neutrophil count, LDH, absolute lymphocyte count, total lymphocyte count, and serum albumin level were mostly used to compute the NLR (absolute neutrophil count divided by absolute lymphocyte count $)$ and the PNI $(10 \times$ serum albumin value, $g /$ $\mathrm{dL}+0.005 \times$ total lymphocyte count $/ \mathrm{mm}^{3}$ ).

\section{Study assessments}

Drug efficacy was assessed every 8-12 weeks based on the Response Evaluation Criteria in Solid Tumors (RECIST, version 1.1) by computed tomography (CT) scan. The data deadline was August 2019. The Common Terminology 
Criteria for Adverse Events (CTCAE) of the National Cancer Institute (version 4.03) was used to assess patients' adverse events. The irAEs were defined as adverse events that reflected a disorder of the immune system, such as rash, colitis, liver dysfunction, thyroid disorder, and other conditions. The irAEs were evaluated for 3 months, because the incidence of irAEs has been reported to be highest within the first 12 weeks [20].

Progression-free survival (PFS) was defined as the first day of immunotherapy to the date of disease progression. Overall survival (OS) was from the time of initial anti-PD-1 immunotherapy to death from any cause or last follow-up.

\section{Statistical analysis}

Patients were stratified as low- and high-NLR $(<5$ and $\geq 5$, respectively) [10], low- and high-LDH ( $<240$ and $\geq 240$ U/L) [12], or low- and high-PNI ( $<45$ and $\geq 45$ ) groups [21]. These cutoffs were selected according to literature references $10,12,21$ and their median, respectively.

PFS and OS curves were calculated using the Kaplan-Meier method, and the log-rank test was employed to assess differences. Cox regression models were applied to find independent indicators associated with PFS and OS. Factors which were statistically significant in the univariate analysis were incorporated into the multivariate analysis. Logistic regression analysis was applied to explore the correlation between peripheral blood markers and the onset of irAEs. SPSS 25.0 software (SPSS, Chicago, IL) was used for all the statistical tests. A $p$ value $<0.05$ was considered statistically significant.

\section{Results}

\section{Patient characteristics}

In our study, 102 patients were enrolled who accepted at least four cycles of immunotherapy (Table 1). Every patient was administered monotherapy with PD-1 inhibitor; 19 patients accepted PD-1 inhibitors as first-line treatment. The median age was 62 years. Most were men (87/102, 85.3\%); most had no or undetected sensitive gene mutations $(94 / 102$, 92.2\%); and most had an ECOG performance status of 0-1 $(89 / 102,87.3 \%)$.

\section{Univariate and multivariate analyses of biomarkers for OS and PFS}

For the population overall, the median OS and PFS were 9 months and 3.7 months, respectively. According to the univariate analysis, the high-NLR group had a significantly worse median OS (3.7 months) and median PFS
Table 1 Patient characteristics

\begin{tabular}{|c|c|c|}
\hline Feature & $\mathrm{N}$ & Percentage $(\%)$ \\
\hline Gender & 102 & 100 \\
\hline Female & 15 & 14.7 \\
\hline Male & 87 & 85.3 \\
\hline \multicolumn{3}{|l|}{ Age (years) } \\
\hline$<60$ & 39 & 38.2 \\
\hline$\geq 60$ & 63 & 61.8 \\
\hline \multicolumn{3}{|l|}{ Histology } \\
\hline Adenocarcinoma & 58 & 56.9 \\
\hline Squamous carcinoma & 42 & 41.2 \\
\hline Large cell carcinoma & 2 & 1.9 \\
\hline \multicolumn{3}{|l|}{ Clinical stage } \\
\hline IIIB & 40 & 39.2 \\
\hline IV & 62 & 60.8 \\
\hline \multicolumn{3}{|l|}{ ECOG PS } \\
\hline $0-1$ & 89 & 87.3 \\
\hline 2 & 13 & 12.7 \\
\hline \multicolumn{3}{|l|}{ Smoking status } \\
\hline Never & 41 & 40.2 \\
\hline Former/current & 61 & 59.8 \\
\hline \multicolumn{3}{|l|}{ Line of immunotherapy } \\
\hline First & 19 & 18.6 \\
\hline Second & 51 & 50.0 \\
\hline$\geq$ Third & 32 & 31.4 \\
\hline \multicolumn{3}{|l|}{ Actionable mutation } \\
\hline$(-) /$ undetected & 94 & 92.2 \\
\hline EGFR or ALK/ROS1(+) & 8 & 7.8 \\
\hline \multicolumn{3}{|l|}{ Number of metastatic sites } \\
\hline$<3$ & 46 & 45.1 \\
\hline$\geq 3$ & 56 & 54.9 \\
\hline \multicolumn{3}{|l|}{ Immune adverse events } \\
\hline No & 63 & 61.8 \\
\hline Yes & 39 & 38.2 \\
\hline \multicolumn{3}{|l|}{ Type of immunotherapy } \\
\hline Nivolumab & 11 & 10.8 \\
\hline Pembrolizumab & 26 & 25.5 \\
\hline Toripalimab & 30 & 29.4 \\
\hline Sintilimab & 35 & 34.3 \\
\hline
\end{tabular}

ECOG PS Eastern Cooperative Oncology Group performance status, $E G F R$ epidermal growth factor receptor, $A L K$ anaplastic lymphoma kinase, ROS1 c-ros oncogene 1

(3.2 months) compared with the low-NLR group (9.8 months and 7.3 months, respectively; Table 2). The high-LDH group had a significantly worse median OS (8.0 months) and median PFS (3.4 months) compared with the low-NLR group (14.6 months and 12.3 months). The high-PNI group had a significantly better median OS (11.5 months) and median PFS (6.3 months) compared with the low-PNI group (4.2 months and 3.3 months). The multivariate analysis 


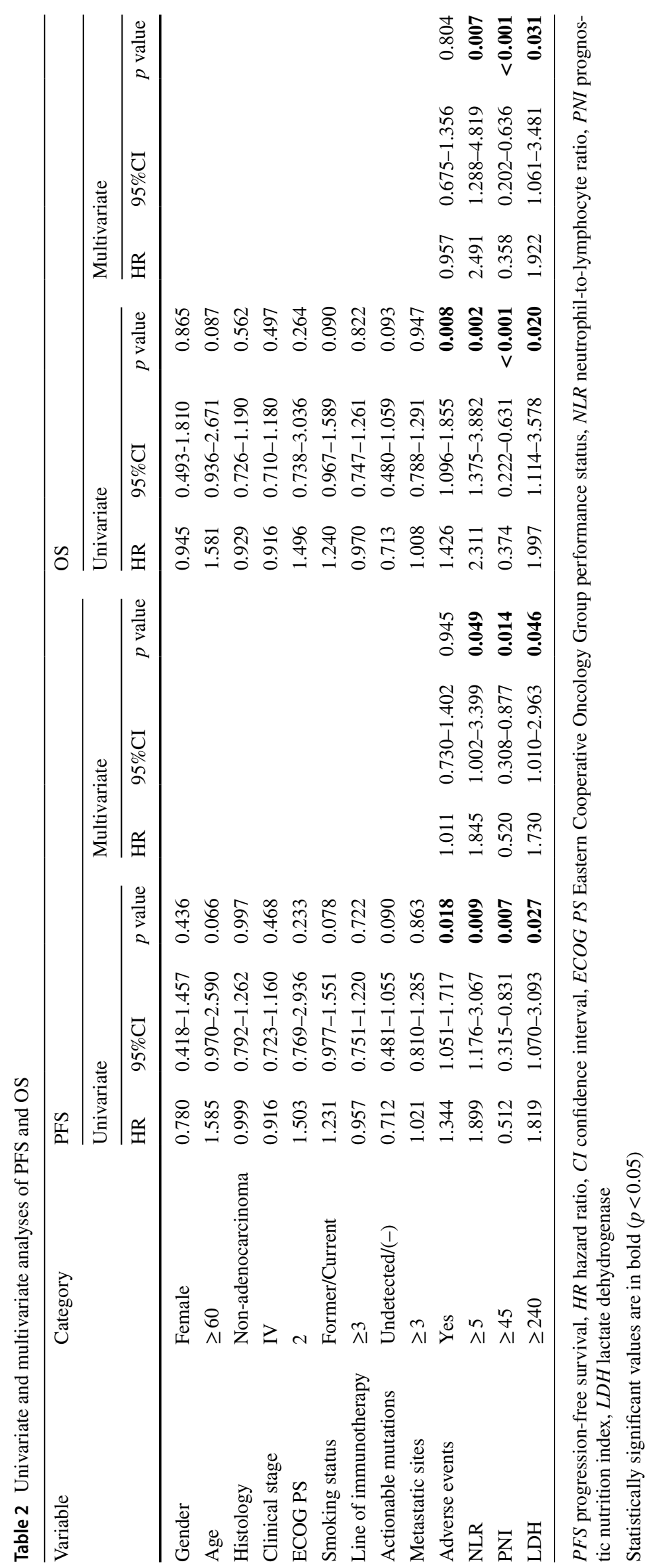


showed that the following factors were significantly associated with OS and PFS (Table 2): NLR $\geq 5, \mathrm{LDH} \geq 240 \mathrm{U} / \mathrm{L}$, and PNI $\geq 45$ (Fig. 1).

\section{Multifactor model for survival outcome of patients treated with PD-1 inhibitors}

We explored the OS and PFS by the number of advantaged factors (i.e., $\mathrm{NLR}<5, \mathrm{LDH}<240 \mathrm{U} / \mathrm{L}$, and $\mathrm{PNI} \geq 45$;
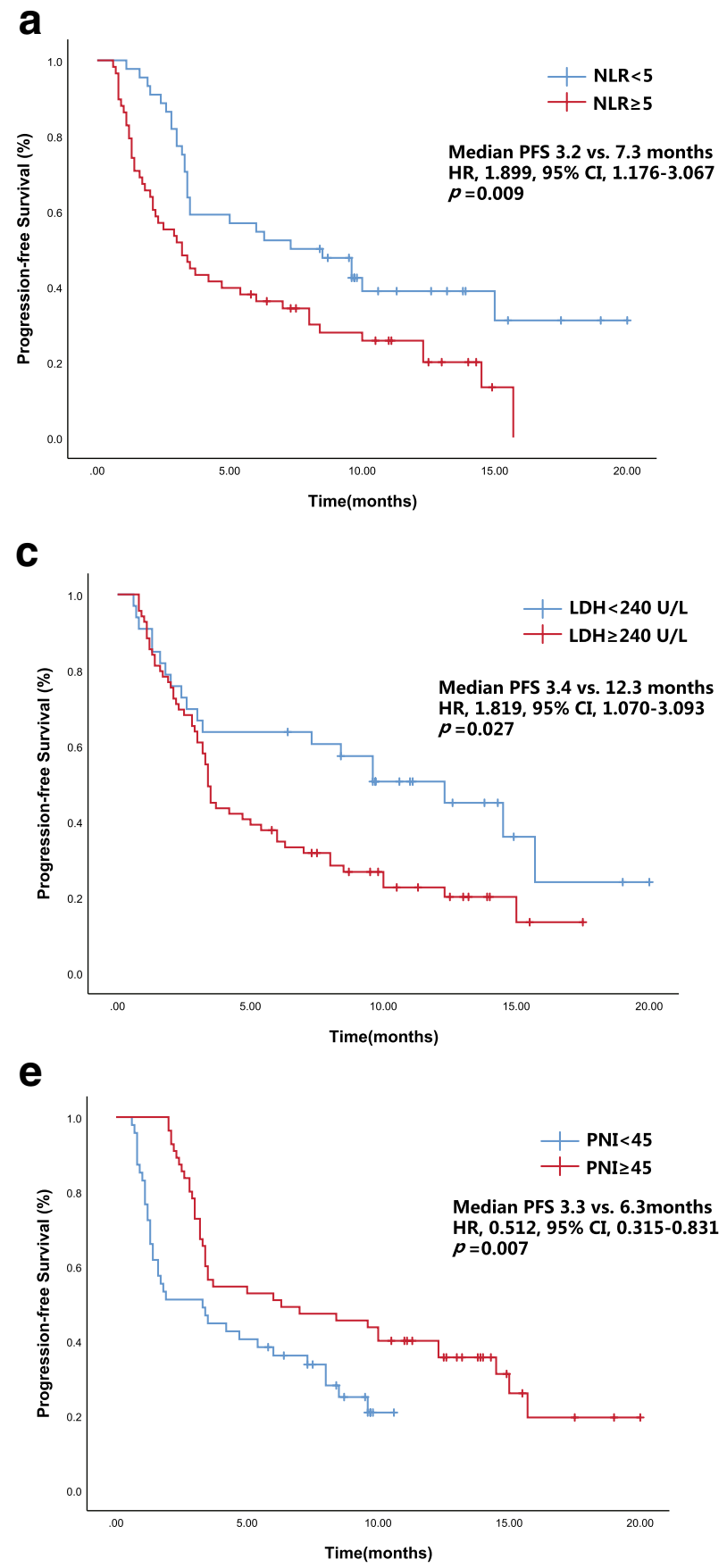

Fig. 2). Of the 102 patients, 24 (23.5\%) had no favorable factors (group D, in Fig. 2). The OS and PFS of this group were significantly shorter than that of patients with 1,2 , or 3 of the favorable factors (groups $\mathrm{C}, \mathrm{B}$, and A; $p<0.001$ ).

\section{Immune-related adverse events (irAEs)}

Thirty-nine patients (38.2\%) experienced, in all, 6 different irAEs of any grade, and 6 patients (5.8\%) experienced

\section{b}
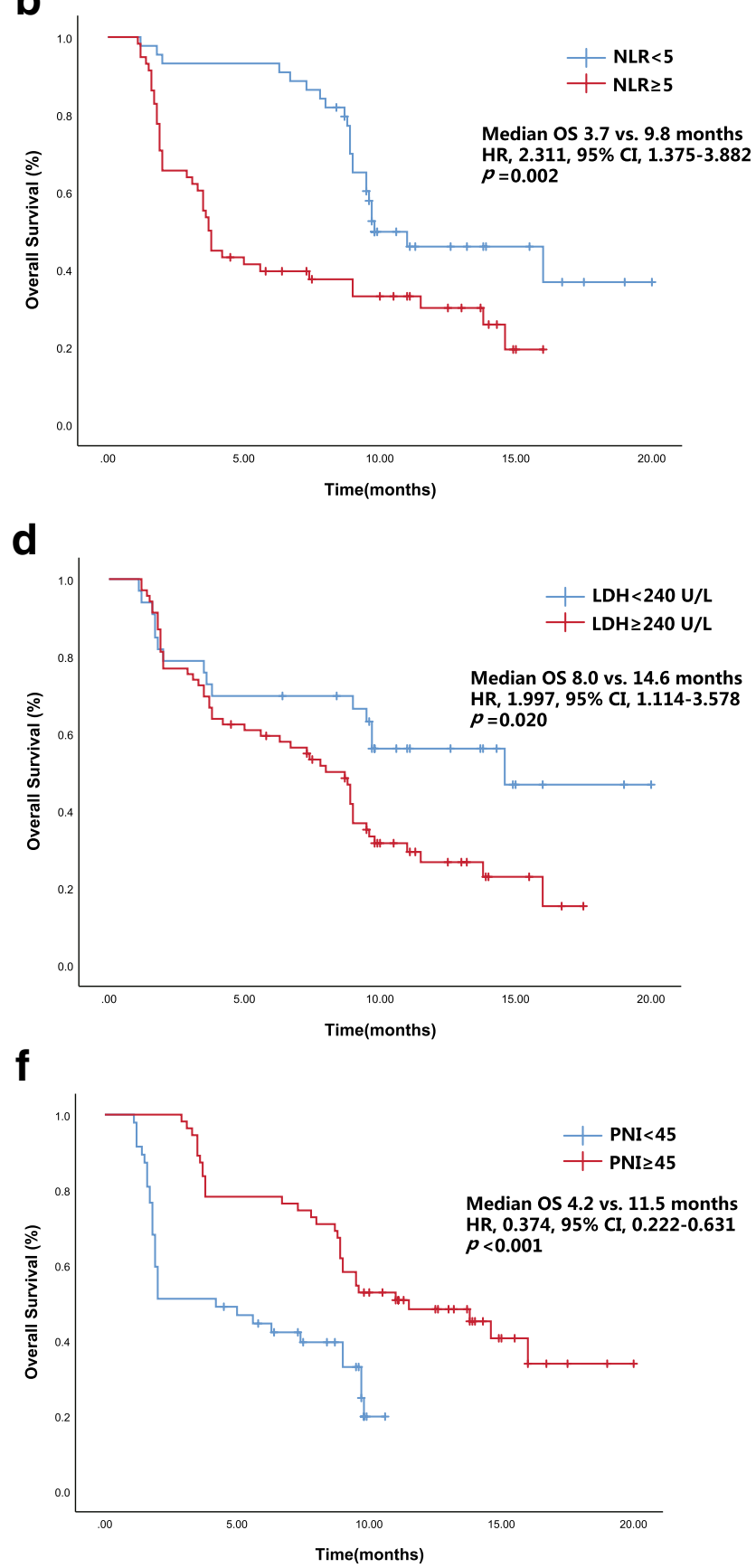

Fig. 1 PFS (a, $, \mathbf{e}, \mathbf{e})$ and OS (b, d, f) curves of patients stratified according to peripheral blood markers (NLR, LDH, and PNI) 

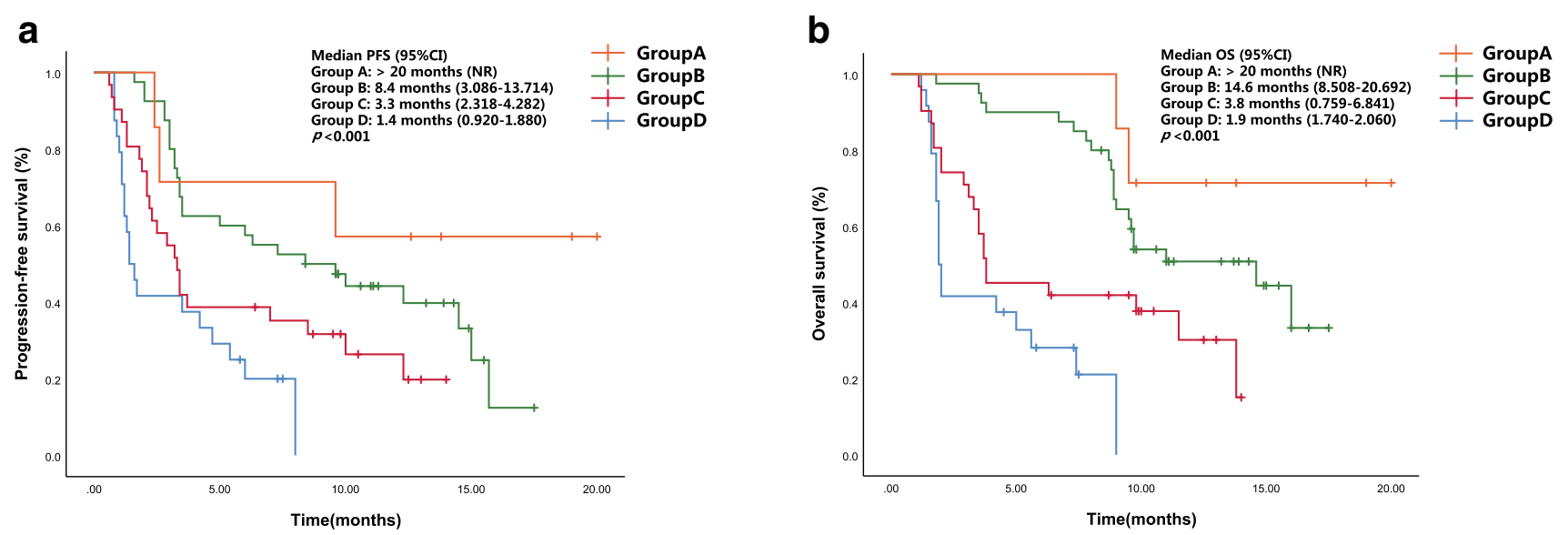

Fig. 2 PFS (a) and OS (b) curves of the multifactor model according to the number of advantageous factors at baseline (NLR $<5$, LDH $<240$ $\mathrm{U} / \mathrm{L}$, and $\mathrm{PNI} \geq 45$ ). Abbreviation: NR, not reached

high-grade irAEs. The commonly seen irAEs (any grade) were $\operatorname{rash}(n=13,33.3 \%)$, liver dysfunctions $(n=9,23.1 \%)$, and hypothyroidism $(n=7,17.9 \%)$. The most common severe irAEs $($ grade $\geq 3)$ were $\operatorname{rash}(n=2,5.1 \%)$. The median OS of the 39 patients with irAEs (11.5 months) was significantly preferable to that of the 63 patients without irAEs (4.2 months; Table 2, Fig. 3). Similarly, the median PFS of the patients with irAEs (9.6 months) was significantly better than that of patients without irAEs (3.4 months). However, irAEs were not an independent prognostic risk factor of OS and PFS in the multivariate analysis.

The low-NLR, low-LDH, and high-PNI groups consisted of 32 (72.7\%), 15 (45.5\%), and 30 (54.5\%) patients, respectively (Table 3 ). The univariate analysis indicated associations between low-NLR or high-PNI and any grade of irAEs ( $p<0.001$, both). The multivariate logistic regression analysis showed that high PNI $(p=0.001)$ and low
NLR $(p<0.001)$ were independent predictors for the onset of irAEs.

\section{Discussion}

Although the preciseness of lung cancer treatment has improved significantly in recent years, NSCLC remains challenging. The emergence of PD-1 inhibitors has brought hope to patients with advanced NSCLC, but many clinical studies have shown that no more than $20 \%$ of patients benefit. Therefore, effective predictive biomarkers are urgently needed for screening potential beneficial groups.

PD-L1 is highly expressed on the cell membranes of NSCLC. Anti-PD-1 immunotherapy of NSCLC is designed to block the signal between PD-1 on T cells and PD-L1 on tumor cells [22]. Graves et al. [23] reported that the PD-1 a

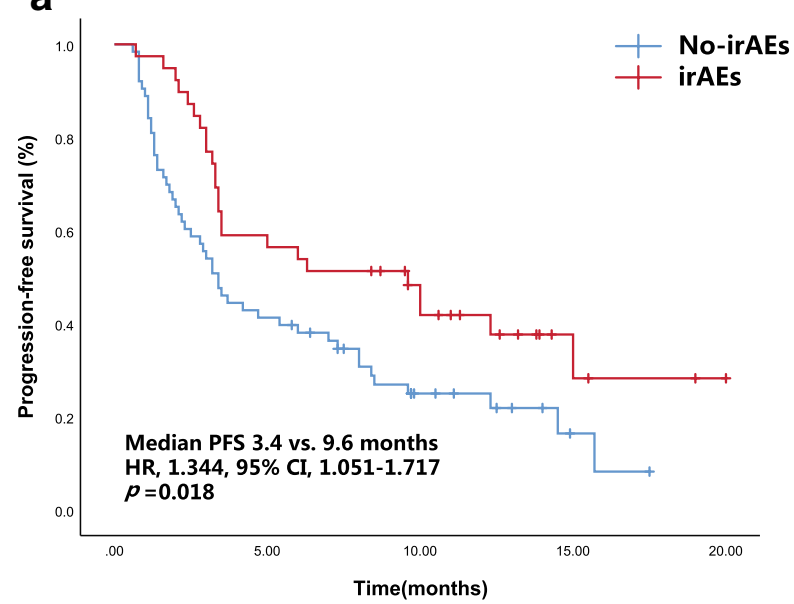

b

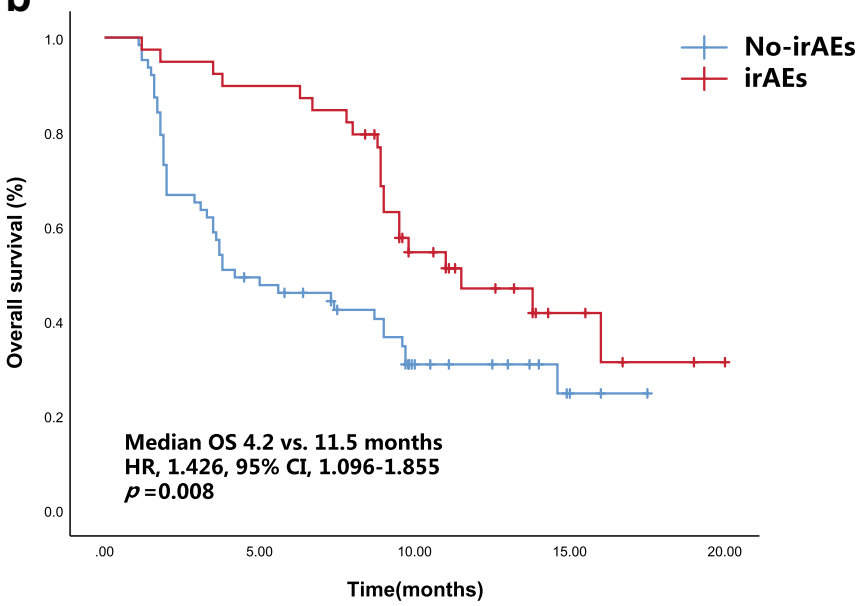

Fig. 3 PFS (a) and OS (b) curves of patients according to the onset of irAEs 
Table 3 Levels of the peripheral blood markers by irAE development

\begin{tabular}{|c|c|c|c|c|c|}
\hline \multirow[t]{2}{*}{ Blood parameter } & \multirow[t]{2}{*}{ irAEs, $n(\%)$} & \multicolumn{2}{|l|}{ Univariate } & \multicolumn{2}{|l|}{ Multivariate } \\
\hline & & OR $(95 \% \mathrm{CI})$ & $p$ value & OR $(95 \% \mathrm{CI})$ & $p$ value \\
\hline L-NLR $(n=44)$ & $32(72.7)$ & $0.051(0.02-0.14)$ & $<0.001$ & $0.04(0.01-0.13)$ & $<0.001$ \\
\hline H-NLR $(n=58)$ & $7(12.1)$ & 1 & & 1 & \\
\hline L-LDH $(n=33)$ & $15(45.5 \%)$ & $0.64(0.28-1.49)$ & 0.301 & $0.45(0.14-1.41)$ & 0.169 \\
\hline H-LDH $(n=69)$ & $24(34.8 \%)$ & 1 & & 1 & \\
\hline H-PNI $(n=55)$ & $30(54.5 \%)$ & $5.07(2.06-12.4)$ & $<0.001$ & $7.61(2.20-26.3)$ & 0.001 \\
\hline L-PNI $(n=47)$ & $9(19.1 \%)$ & 1 & & 1 & \\
\hline
\end{tabular}

$O R$ odds ratio, $C I$ confidence interval, $H$-NLR high NLR, $H$ - $L D H$ high LDH, $H$-PNI high PNI, irAEs immune-related adverse events, $N L R$ neutrophil-to-lymphocyte ratio, $L D H$ lactate dehydrogenase, $P N I$ prognostic nutrition index, $L-N L R$ low NLR, $L-L D H$ low $L D H, L-P N I$ low PNI level on CD4+ T cells in the blood of melanoma patients who responded to anti-PD-1 therapy was higher than that of non-responders. Currently, the PD-L1 level is a commonly used marker for predicting the efficacy of immunotherapy. As reported by CheckMate-057 [24] and Keynote-010 [25], patients with high PD-L1 levels in tumor tissues, and who received PD-1/PD-L1 inhibitors, had better survival outcomes compared with those who were not given this treatment. Nevertheless, CheckMate-017 [26] reported that patients who were PD-L1-negative also responded well. Therefore, PD-L1 level is not sufficient as the sole decisive predictor of immunotherapy. TMB is another potential predictive biomarker that has received much attention, but has been considered only as a reference marker; TMB should be explored further in clinical research. In May 2017, pembrolizumab received approval by the United States Food and Drug Association for the treatment of metastatic or advanced solid tumors with mismatch repair deficiency (i.e., high levels of microsatellite instability, or MSI-H). However, the American Society of Clinical Oncology (ASCO) reported in 2016 that MSI-H occurs in only $0.4-0.8 \%$ of lung cancer. The predictive markers discussed above are limited by cumbersome detection protocols and high cost. Hence, it is necessary to explore for markers that can effectively predict the benefit of therapy, but which are also clinically practical and without serious drug toxicity.

It has been reported that nutritional status and inflammatory status have prognostic relevance in patients with a variety of cancers [27, 28]. The markers evaluated in the present study (NLR, LDH, and PNI) reflect well the inflammation and nutritional status. The association between baseline NLR and the prognosis of melanoma patients treated with immune checkpoint inhibitors has been demonstrated [29, 30]. Bagley et al. [10] studied 175 patients with advanced NSCLC treated with nivolumab and concluded that NLR $\geq 5$ at baseline was a risk factor of inferior OS (HR 1.83, 95\% CI 1.2-2.8; $p=0.006$ ) and inferior PFS (HR 1.42, 95\% CI 1.02-2.0; $p=0.04$ ), compared with $\mathrm{NLR}<5$. In the multivariate analysis, NLR $\geq 5$ was also independently linked to worse outcomes. In addition, another retrospective study showed that baseline NLR $>5$ was associated with poor OS [31]. In the present study, we also concluded that an NLR of 5 was the optimal cutoff: NLR $\geq 5$ was associated with worse OS (HR 2.311,95\% CI 1.375-3.882; $p=0.002)$ and PFS (HR 1.899, 95\% CI 1.176-3.067; $p=0.009)$. Furthermore, NLR $\geq 5$ was an independent prognostic risk indictor in the multivariate analysis model.

$\mathrm{LDH}$ is produced by rapidly growing tumors and therefore reflects the tumor burden. LDH was found associated with outcomes in melanoma patients treated with immune checkpoint inhibitors [32, 33]. Several studies used LDH to forecast PFS [12, 34] and OS [6, 11] in patients with NSCLC treated with immune checkpoint inhibitors. Taniguchi et al. [12] discovered that among patients with advanced NSCLC treated with nivolumab, those with baseline LDH > $240 \mathrm{U} / \mathrm{L}$ had a significantly worse PFS compared with those with LDH $\leq 240$ U/L. Similarly, the present analysis showed that a baseline $\mathrm{LDH} \geq 240 \mathrm{U} / \mathrm{L}$ was associated with worse OS and PFS.

In 1984, Japanese scholars Onodera et al. [35] proposed a simplified version of the PNI, which is based only on serum albumin and total the lymphocyte count. Serum albumin concentration can indicate the nutritional status of patients and chronic inflammation. In addition to reflected inflammatory status, the lymphocyte count is also an important parameter of the immune function [36]. Several studies have reported a significant association between the PNI and the prognosis of patients with a variety of malignant cancers. However, there has been little research regarding the value of the PNI in cancer immunotherapy. In the present study, a pretreatment $\mathrm{PNI} \geq 45$ was associated with better OS (HR $0.374,95 \%$ CI $0.222-0.631 ; p<0.001)$ and PFS (HR 0.512, 95\% CI 0.315-0.831; $p=0.007)$ compared with PNI < 45. In the multivariate analysis, $\mathrm{PNI} \geq 45$ was also independently associated with worse PFS and OS. An evaluation of baseline PNI may provide meaningful information for selecting suitable patients in immunotherapy. 
The increasing use of immune checkpoint inhibitors has been accompanied by a rise in unique adverse events, known as irAEs, which can lead to troubling morbidity and treatment discontinuations [37]. Nakaya et al. [38] reported that patients with NSCLC treated with nivolumab and who experienced an associated irAEs had better PFS compared with those who had no irAEs. Similarly, the present analysis showed that the patients who suffered from irAEs had longer PFS and OS, although this did not rise to the level of an independent prognosis marker for PFS and OS. Moreover, the rate of irAEs in our research also resembled the study that reported by Nakaya et al. [38]. The current study also explored an association between irAEs and the peripheral blood markers NLR, LDH, and PNI and found that low NLR and high PNI were significantly associated with the onset of irAEs. So, the baseline NLR and PNI may be used as a convenient tool to identify irAEs timely, which is essential for reducing the risk of hospitalization and the costs of treatment.

The limitations of this research are its retrospective nature and relatively small sample size. Although the results of our study are interesting, the predictive value of the peripheral blood markers (NLR, LDH, and PNI) on PFS, OS or irAEs requires further validation by randomized studies with an untreated control group. Furthermore, most of the patients received PD-1 inhibitors as their second-line treatment or beyond. Thus, the degree of baseline inflammation may be affected by previous treatments, although the number of lines of immunotherapy was not associated with clinical outcomes. Additionally, the PD-L1 status was known in so few patients that we could not include in our study and was conducted using different methods. Finally, immune-related response criteria were not applied in this study, because it was not a prospective research and most physicians are unfamiliar with the criteria. Despite these limitations, a unique aspect of this study was the combined model of three baseline peripheral blood markers for the outcome of PD-1 inhibitors. In addition, to our best knowledge, it is the first study to explore a correlation between peripheral blood markers (LDH and PNI) and irAEs in patients with advanced NSCLC accepting PD-1 inhibitors, or an evaluation of the effect of irAEs on both PFS and OS.

In conclusion, the pretreatment peripheral blood markers analyzed herein (NLR, LDH, and PNI) may correlate with outcomes and the onset of irAEs in patients with advanced NSCLC accepting PD-1 inhibitors. This provides some directions for clinical research on immunotherapy of lung cancer. With the rising attention to health-related costs and precision medicine, the predictive role of peripheral blood markers in cancer immunotherapy is very meaningful. These preliminary results warrant further research.
Author contributions LP, YW, FL, XQ, XZ, CF, and XQ contributed to the acquisition, analysis and interpretation of data, and statistical analysis and drafting of the manuscript. LP, YW, and YL were responsible for the study concept and design, analysis and interpretation of the data, drafting of the manuscript, and obtaining funding and study supervision. All authors read and approved the final manuscript.

Funding This study was supported by Grants from the National Natural Science Foundation of China (Nos. 81560379, 81460292, 81660315), the Surface Project of the Natural Science Foundation of Jiangxi Province (No. 20181BAB205046), the Technology Supporting Program of Jiangxi Province (No. 2015BBG70236), the Key Project of the Education Department of Jiangxi Province (No. GJJ170012), and the Guiding Science and Technology Project of Ganzhou (No. GZ2018ZSF306).

\section{Compliance with ethical standards}

Conflict of interest The authors declare that they have no conflict of interest.

Ethical approval and ethical standards The study was performed in accordance with the Declaration of Helsinki.

Informed consent All patients from First Affiliated Hospital of Nanchang University provided signed informed consent for the treatments performed and for the anonymous use of their clinical data for research and publication purposes.

Open Access This article is licensed under a Creative Commons Attribution 4.0 International License, which permits use, sharing, adaptation, distribution and reproduction in any medium or format, as long as you give appropriate credit to the original author(s) and the source, provide a link to the Creative Commons licence, and indicate if changes were made. The images or other third party material in this article are included in the article's Creative Commons licence, unless indicated otherwise in a credit line to the material. If material is not included in the article's Creative Commons licence and your intended use is not permitted by statutory regulation or exceeds the permitted use, you will need to obtain permission directly from the copyright holder. To view a copy of this licence, visit http://creativecommons.org/licenses/by/4.0/.

\section{References}

1. Chen W, Zheng R, Baade PD, Zhang S, Zeng H, Bray F, Jemal A, Yu XQ, He J (2016) Cancer statistics in China, 2015. CA Cancer J Clin 66:115-132. https://doi.org/10.3322/caac.21338

2. Gettinger SN, Horn L, Gandhi L et al (2015) Overall survival and long-term safety of nivolumab (anti-programmed death 1 antibody, BMS-936558, ONO-4538) in patients with previously treated advanced non-small-cell lung cancer. J Clin Oncol 33:2004-2012. https://doi.org/10.1200/JCO.2014.58.3708

3. Iwai Y, Hamanishi J, Chamoto K, Honjo T (2017) Cancer immunotherapies targeting the PD-1 signaling pathway. J Biomed Sci 24:26. https://doi.org/10.1186/s12929-017-0329-9

4. Philip M, Rowley DA, Schreiber H (2004) Inflammation as a tumor promoter in cancer induction. Semin Cancer Biol 14:433439. https://doi.org/10.1016/j.semcancer.2004.06.006

5. Ferrucci PF, Ascierto PA, Pigozzo J et al (2018) Baseline neutrophils and derived neutrophil-to-lymphocyte ratio: prognostic relevance in metastatic melanoma patients receiving ipilimumab. Ann Oncol 29:524. https://doi.org/10.1093/annonc/mdx059 
6. Capone M, Giannarelli D, Mallardo D et al (2018) Baseline neutrophil-to-lymphocyte ratio (NLR) and derived NLR could predict overall survival in patients with advanced melanoma treated with nivolumab. J Immunother Cancer 6:74. https://doi.org/10.1186/ s40425-018-0383-1

7. Fujisawa Y, Yoshino K, Otsuka A et al (2018) Baseline neutrophil to lymphocyte ratio combined with serum lactate dehydrogenase level associated with outcome of nivolumab immunotherapy in a Japanese advanced melanoma population. Br J Dermatol 179:213-215. https://doi.org/10.1111/bjd.16427

8. Soyano AE, Dholaria B, Marin-Acevedo JA et al (2018) Peripheral blood biomarkers correlate with outcomes in advanced nonsmall cell lung cancer patients treated with anti-PD-1 antibodies. J Immunother Cancer 6:129. https://doi.org/10.1186/s4042 5-018-0447-2

9. Diem S, Schmid S, Krapf M, Flatz L, Born D, Jochum W, Templeton AJ, Fruh M (2017) Neutrophil-to-lymphocyte ratio (NLR) and platelet-to-lymphocyte ratio (PLR) as prognostic markers in patients with non-small cell lung cancer (NSCLC) treated with nivolumab. Lung Cancer 111:176-181. https://doi. org/10.1016/j.lungcan.2017.07.024

10. Bagley SJ, Kothari S, Aggarwal C et al (2017) Pretreatment neutrophil-to-lymphocyte ratio as a marker of outcomes in nivolumab-treated patients with advanced non-small-cell lung cancer. Lung Cancer 106:1-7. https://doi.org/10.1016/j.lungc an.2017.01.013

11. Mezquita L, Auclin E, Ferrara R et al (2018) Association of the lung immune prognostic index with immune checkpoint inhibitor outcomes in patients with advanced non-small cell lung cancer. JAMA Oncol 4:351-357. https://doi.org/10.1001/ jamaoncol.2017.4771

12. Taniguchi Y, Tamiya A, Isa SI et al (2017) Predictive factors for poor progression-free survival in patients with non-small cell lung cancer treated with nivolumab. Anticancer Res 37:58575862. https://doi.org/10.21873/anticanres.12030

13. Kataoka Y, Hirano K, Narabayashi T, Hara S, Fujimoto D, Tanaka T, Ebi N, Tomii K, Yoshioka H (2018) Carcinoembryonic antigen as a predictive biomarker of response to nivolumab in non-small cell lung cancer. Anticancer Res 38:559-563. https ://doi.org/10.21873/anticanres.12259

14. Buzby GP, Mullen JL, Matthews DC, Hobbs CL, Rosato EF (1980) Prognostic nutritional index in gastrointestinal surgery. Am J Surg 139:160-167

15. Hong S, Zhou T, Fang W et al (2015) The prognostic nutritional index (PNI) predicts overall survival of small-cell lung cancer patients. Tumour Biol 36:3389-3397. https://doi.org/10.1007/ s13277-014-2973-y

16. Okada S, Shimada J, Kato D, Tsunezuka H, Teramukai S, Inoue M (2017) Clinical significance of prognostic nutritional index after surgical treatment in lung cancer. Ann Thorac Surg 104:296-302. https://doi.org/10.1016/j.athoracsur.2017.01.085

17. Eo WK, Chang HJ, Suh J et al (2015) The prognostic nutritional index predicts survival and identifies aggressiveness of gastric cancer. Nutr Cancer 67:1260-1267. https://doi. org/10.1080/01635581.2015.1082112

18. Tokunaga R, Sakamoto Y, Nakagawa S, Miyamoto Y, Yoshida N, Oki E, Watanabe M, Baba H (2015) Prognostic nutritional index predicts severe complications, recurrence, and poor prognosis in patients with colorectal cancer undergoing primary tumor resection. Dis Colon Rectum 58:1048-1057. https://doi. org/10.1097/DCR.0000000000000458

19. Rigamonti A, Imbesi F, Silvani A et al (2019) Prognostic nutritional index as a prognostic marker in glioblastoma: data from a cohort of 282 Italian patients. J Neurol Sci 400:175-179. https ://doi.org/10.1016/j.jns.2019.04.002

20. Haanen J, Carbonnel F, Robert C, Kerr KM, Peters S, Larkin J, Jordan K, Committee EG (2017) Management of toxicities from immunotherapy: ESMO Clinical Practice Guidelines for diagnosis, treatment and follow-up. Ann Oncol 28:iv119-iv42. https://doi.org/10.1093/annonc/mdx225

21. Watanabe I, Kanauchi N, Watanabe H (2018) Preoperative prognostic nutritional index as a predictor of outcomes in elderly patients after surgery for lung cancer. Jpn J Clin Oncol 48:382387. https://doi.org/10.1093/jjco/hyy014

22. Pardoll DM (2012) The blockade of immune checkpoints in cancer immunotherapy. Nat Rev Cancer 12:252-264. https:// doi.org/10.1038/nrc3239

23. Graves M, CelliMarchett G, van Zyl B, Tang D, Vilain RE, van der Westhuizen A, Bowden NA (2019) Monitoring patient response to pembrolizumab with peripheral blood exhaustion marker profiles. Front Med (Lausanne) 6:113. https://doi. org/10.3389/fmed.2019.00113

24. Borghaei H, Paz-Ares L, Horn L et al (2015) Nivolumab versus docetaxel in advanced nonsquamous non-small-cell lung cancer. N Engl J Med 373:1627-1639. https://doi.org/10.1056/NEJMo a1507643

25. Herbst RS, Baas P, Kim DW et al (2016) Pembrolizumab versus docetaxel for previously treated, PD-L1-positive, advanced nonsmall-cell lung cancer (KEYNOTE-010): a randomised controlled trial. Lancet 387:1540-1550. https://doi.org/10.1016/ S0140-6736(15)01281-7

26. Brahmer J, Reckamp KL, Baas P et al (2015) Nivolumab versus docetaxel in advanced squamous-cell non-small-cell lung cancer. N Engl J Med 373:123-135. https://doi.org/10.1056/NEJMo a1504627

27. Kos FT, Hocazade C, Kos M, Uncu D, Karakas E, Dogan M, Uncu HG, Ozdemir N, Zengin N (2015) Assessment of prognostic value of "neutrophil to lymphocyte ratio" and "prognostic nutritional index" as a sytemic inflammatory marker in nonsmall cell lung cancer. Asian Pac J Cancer Prev 16:3997-4002. https://doi.org/10.7314/apjcp.2015.16.9.3997

28. Miyazaki T, Sakai M, Sohda M et al (2016) Prognostic significance of inflammatory and nutritional parameters in patients with esophageal cancer. Anticancer Res 36:6557-6562. https ://doi.org/10.21873/anticanres.11259

29. Ferrucci PF, Gandini S, Battaglia A et al (2015) Baseline neutrophil-to-lymphocyte ratio is associated with outcome of ipilimumab-treated metastatic melanoma patients. Br J Cancer 112:1904-1910. https://doi.org/10.1038/bjc.2015.180

30. Zaragoza J, Caille A, Beneton N, Bens G, Christiann F, Maillard H, Machet L (2016) High neutrophil to lymphocyte ratio measured before starting ipilimumab treatment is associated with reduced overall survival in patients with melanoma. Br J Dermatol 174:146-151. https://doi.org/10.1111/bjd.14155

31. Kiriu T, Yamamoto M, Nagano T et al (2018) The time-series behavior of neutrophil-to-lymphocyte ratio is useful as a predictive marker in non-small cell lung cancer. PLoS ONE 13:e0193018. https://doi.org/10.1371/journal.pone.0193018

32. Kelderman S, Heemskerk B, van Tinteren H et al (2014) Lactate dehydrogenase as a selection criterion for ipilimumab treatment in metastatic melanoma. Cancer Immunol Immunother 63:449 458. https://doi.org/10.1007/s00262-014-1528-9

33. Diem S, Kasenda B, Spain L, Martin-Liberal J, Marconcini R, Gore M, Larkin J (2016) Serum lactate dehydrogenase as an early marker for outcome in patients treated with anti-PD-1 therapy in metastatic melanoma. Br J Cancer 114:256-261. https://doi.org/10.1038/bjc.2015.467 
34. Oya Y, Yoshida T, Kuroda H et al (2017) Predictive clinical parameters for the response of nivolumab in pretreated advanced non-small-cell lung cancer. Oncotarget. 8:103117-103128. https ://doi.org/10.18632/oncotarget.21602

35. Onodera T, Goseki N, Kosaki G (1984) Prognostic nutritional index in gastrointestinal surgery of malnourished cancer patients. Nihon Geka Gakkai Zasshi 85:1001-1005

36. Lee YJ, Kim WR, Han J, Han YD, Cho MS, Hur H, Lee KY, Kim NK, Min BS (2016) Prognostic impact of immunonutritional status changes during preoperative chemoradiation in patients with rectal cancer. Ann Coloproctol 32:208-214. https ://doi.org/10.3393/ac.2016.32.6.208

37. Michot JM, Bigenwald C, Champiat $S$ et al (2016) Immunerelated adverse events with immune checkpoint blockade: a comprehensive review. Eur J Cancer 54:139-148. https://doi. org/10.1016/j.ejca.2015.11.016

38. Nakaya A, Kurata T, Yoshioka H et al (2018) Neutrophil-to-lymphocyte ratio as an early marker of outcomes in patients with advanced non-small-cell lung cancer treated with nivolumab. Int J Clin Oncol 23:634-640. https://doi.org/10.1007/s1014 7-018-1250-2

Publisher's Note Springer Nature remains neutral with regard to jurisdictional claims in published maps and institutional affiliations. 